Article

\title{
A Comparison of Estimating Crop Residue Cover from Sentinel-2 Data Using Empirical Regressions and Machine Learning Methods
}

\author{
Yanling Ding ${ }^{1,2} \mathbb{C}^{\text {, Hongyan Zhang }}{ }^{1,2}$, Zhongqiang Wang ${ }^{1}{ }^{\circledR}$, Qiaoyun Xie ${ }^{3 \oplus}$, Yeqiao Wang ${ }^{4, *}$, \\ Lin Liu ${ }^{1}$ and Christopher C. Hall ${ }^{3}$ \\ 1 Key Laboratory of Geographical Processes and Ecological Security in Changbai Mountains, Ministry of \\ Education, School of Geographical Sciences, Northeast Normal University, Changchun 130024, China; \\ dingy1720@nenu.edu.cn (Y.D.); zhy@nenu.edu.cn (H.Z.); wangzq027@nenu.edu.cn (Z.W.); \\ liul769@nenu.edu.cn (L.L.) \\ 2 Urban Remote Sensing Application Innovation Center, School of Geographical Sciences, \\ Northeast Normal University, Changchun 130024, China \\ 3 Faculty of Science, University of Technology Sydney, Sydney, NSW 2007, Australia; \\ qiaoyun.xie@uts.edu.au (Q.X.); Christopher.Hall-1@uts.edu.au (C.C.H.) \\ 4 Department of Natural Resources Science, University of Rhode Island, Kingston, RI 02881, USA \\ * Correspondence: yqwang@uri.edu
}

Received: 29 March 2020; Accepted: 5 May 2020; Published: 6 May 2020

check for updates

\begin{abstract}
Quantifying crop residue cover (CRC) on field surfaces is important for monitoring the tillage intensity and promoting sustainable management. Remote-sensing-based techniques have proven practical for determining CRC, however, the methods used are primarily limited to empirical regression based on crop residue indices (CRIs). This study provides a systematic evaluation of empirical regressions and machine learning (ML) algorithms based on their ability to estimate CRC using Sentinel-2 Multispectral Instrument (MSI) data. Unmanned aerial vehicle orthomosaics were used to extracted ground CRC for training Sentinel-2 data-based CRC models. For empirical regression, nine MSI bands, 10 published CRIs, three proposed CRIs, and four mean textural features were evaluated using univariate linear regression. The best performance was obtained by a three-band index calculated using (B2 - B4)/(B2 - B12), with an $R^{2}{ }_{\mathrm{cv}}$ of 0.63 and $\mathrm{RMSE}_{\mathrm{cv}}$ of $6.509 \%$, using a 10 -fold cross-validation. The methodologies of partial least squares regression (PLSR), artificial neural network (ANN), Gaussian process regression (GPR), support vector regression (SVR), and random forest (RF) were compared with four groups of predictors, including nine MSI bands, 13 CRIs, a combination of MSI bands and mean textural features, and a combination of CRIs and textural features. In general, ML approaches achieved high accuracy. A PLSR model with 13 CRIs and textural features resulted in an accuracy of $R^{2}{ }_{\mathrm{cv}}=0.66$ and $\mathrm{RMSE}_{\mathrm{cv}}=6.427 \%$. An RF model with predictors of MSI bands and textural features estimated CRC with an $R^{2}{ }_{\mathrm{cv}}=0.61$ and $\mathrm{RMSE}_{\mathrm{cv}}=6.415 \%$. The estimation was improved by an SVR model with the same input predictors $\left(R^{2}{ }_{\mathrm{cv}}=0.67, \mathrm{RMSE}_{\mathrm{cv}}=6.343 \%\right)$, followed by a GPR model based on CRIs and textural features. The performance of GPR models was further improved by optimal input variables. A GPR model with six input variables, three MSI bands and three textural features, performed the best, with $R_{\mathrm{cv}}^{2}=0.69$ and $\mathrm{RMSE}_{\mathrm{Cv}}=6.149 \%$. This study provides a reference for estimating CRC from Sentinel-2 imagery using ML approaches. The GPR approach is recommended. A combination of spectral information and textural features leads to an improvement in the retrieval of CRC.
\end{abstract}

Keywords: crop residue cover; crop residue indices; empirical regression; machine learning regression; Sentinel-2 MSI; textural feature; unmanned aerial vehicle 


\section{Introduction}

Crop residues, such as stalks, stems, leaves, and seed pods, are materials left on the surface of agricultural fields after harvest. Crop residue cover (CRC), as an indicator of the amount of crop residue left on the soil surface, plays an important role in evaluating tillage intensity [1,2]. CRC is a key input parameter in models to predict the impact of agricultural systems on soil organic carbon, greenhouse gas emissions, and crop production $[3,4]$. Therefore, it is critical to estimate CRC accurately, thereby enabling the evaluation of the effectiveness of tillage practice and promoting agricultural sustainability.

Currently, the measurement of CRC depends on manual survey-based methods, such as the line-point transect. These methods are time-consuming and labor-intensive, thus making systematic and continuous quantification of CRC over large areas difficult [5,6]. Alternatively, remote sensing is an efficient technique to acquire CRC spatially and temporally in a rapid, accurate, and objective manner [7,8]. Remote sensing techniques used to estimate CRC can be classified into empirical regression based on crop residue indices (CRIs), classification [9,10], spectral unmixing [11], and spectral angle methods $[12,13]$. The most widely used among these methods is empirical regression constructed from a linear or nonlinear relationship between CRC and the CRIs, also known as "the CRI technique".

A series of CRIs have been designed to improve the detection of CRC. One such CRI, cellulose absorption index (CAI), was developed by Nagler et al. [14] based on a unique absorption feature associated with cellulose and lignin at $2100 \mathrm{~nm}$ in the spectra of crop residue. The CAI has proven very accurate in quantifying CRC [15]. However, its application is limited because hyperspectral imagery is currently only available on airborne or proximal platforms. Various broadband indices have been developed for mapping CRC using multispectral satellite imagery, such as the Advanced Spaceborn Thermal Emission and Reflectance Radiometer (ASTER) and the Landsat family. The well-known ASTER CRIs include the lignin cellulose absorption index (LCA), and the shortwave infrared (SWIR) normalized difference residue index (SINDRI) calculated from SWIR bands [16,17], which are effective at measuring CRC due to the precise measurement of cellulose and lignin absorption features [18]. The CRIs developed from the Landsat imageries are also primarily dependent on SWIR bands, such as normalized difference tillage index (NDTI) [19], normalized difference indices (NDI, NDI5, NDI7) [20], normalized difference senescent vegetation index (NDSVI) [21], and normalized difference residue index (NDRI) [22]. Previous studies showed that NDTI performed the best in most cases [15], and NDRI showed an advantage in minimizing the effects of green vegetation [22]. Most of the above indices are limited to normalized difference ratios of two bands. Index formulations, e.g., simple ratio and normalized difference ratio, and band selection have impacts on the performance of the estimation [23]. It is likely that indices based on more than two bands and different formulations would lead to further improvements in the retrieval of variables from remote sensing imagery.

The Sentinel-2 satellites launched in 2015 and 2017 carry Multi-spectral Instruments (MSI) with 13 spectral bands. Apart from the three red-edge bands, the MSI bands have corresponding spectrum regions to the Landsat 8 Operational Land Imager (OLI). Moreover, Sentinel-2 data possess finer spatial and temporal resolutions, which is more suitable for agricultural monitoring. However, there is a lack of studies on evaluating the ability of Sentinel-2 data in CRC estimation [4,24].

Besides the empirical regression approach, various machine learning (ML) regression algorithms have been developed and are popular in bio-geophysical variable retrieval due to their computational efficiency and effectiveness $[25,26]$. ML algorithms have shown advantages in capturing the non-linear relationship of input features and retrieval targets and can be massively multivariate, involving several variables. Commonly used ML methods include artificial neural network (ANN), support vector machine (SVM), Gaussian processes regression (GPR) [27-29]. To date, very few studies have investigated the use of $\mathrm{ML}$ regression for the prediction of CRC [30]. Therefore, evaluating the performance of ML regressions in CRC estimation is urgently needed. CRIs that are designed to enhance the discrimination of crop residue from soil are reportedly more correlated to CRC than spectral reflectances [31]. Therefore, it is worth exploring the use of CRIs as input features in the ML models for the prediction of CRC. Furthermore, several studies reported that textural features 
were useful in estimating vegetation parameters (i.e., leaf area index (LAI)) and combining both textural features and spectral information provided much improvement compared with using spectral information only [32-34]. In addition, Jin et al. [35] evaluated three processing techniques to estimate CRC: regressions based on CRIs, textural features, and combinations of CRIs with textural analyses. They found that textural features were correlated with CRC and the regression based on a combination of textural features and CRIs yields a better result than the other two approaches. Based on these studies, it is necessary to evaluate the potential of textural features used as input predictors in the machine learners for the prediction of CRC.

To address the gaps, this study focused on evaluating the potential of Sentinel-2 data on CRC estimation using an empirical regression approach and ML regression techniques. We hypothesize that using different combinations of MSI bands, CRIs, and textural features could provide improved estimates of CRC than using any single source feature alone. Therefore, we evaluated and compared the performance of univariate regression against individual MSI bands, CRIs and texture features, as well as the retrieval accuracies of partial least squares regression (PLSR), ANN, support vector regression (SVR), GPR, and random forest (RF) associated with MSI bands, CRIs, and their combinations with textural features.

\section{Materials and Methods}

\subsection{Study Area}

The study area is located in Changchun $\left(43^{\circ} 5^{\prime} \mathrm{N}-45^{\circ} 15^{\prime} \mathrm{N}, 124^{\circ} 18^{\prime} \mathrm{E}-127^{\circ} 2^{\prime} \mathrm{E}\right)$, Jilin province, Northeast China (Figure 1). The region is situated in a temperate continental climate zone with four seasons, characterized by a hot and rainy summer and cold and dry winter. The test area is about $320 \times 450 \mathrm{~m}$. Maize is the main crop in this region. Local maize cultivars are planted and harvested in May and October, respectively. The variability of in-ground CRC is due to the different harvesting traditions that local farmers have been implemented.

\subsection{Unmanned Aerial Vehicle Imagery}

Field CRC measurements were obtained with an unmanned aerial vehicle (UAV) on 29 March 2018. We used a DJI Inspire 1 quadcopter (DJI, Shenzhen, China). The UAV carried a high resolution digital camera with a 1/2.3" CMOS sensor and 12.4 million effective pixels to generate images with red, green, and blue (RGB) channels. At $100 \mathrm{~m}$ flight height, the captured 537 images, retaining horizontal and vertical overlaps of at least $80 \%$ and $75 \%$, respectively, and possessing a spatial resolution of $4.4 \mathrm{~cm}$. The Pix4D software (Pix4D SA, Lausanne, Switzerland) and ENVI 5.3 were applied for image mosaicking and georectification with 12 ground control points obtained from Google Earth as the reference.

\subsection{Sentinel-2 Data}

We used Sentinel-2B images acquired on March 27, 2018. The images were downloaded from the Copernicus Open Access Hub website (https://scihub.copernicus.eu/). Atmospheric correction was done using Sen2cor (v.2.4.0) software. The $20 \mathrm{~m}$ spatial resolution images, consisting of B2, B3, B4, B5, B6, B7, B8a, B11 and B12, were used to calculate CRIs (Table 1), and the $10 \mathrm{~m}$ spatial resolution images, including B2, B3, B4, and B8, were used to extract textural features for the high spatial resolution.

\subsection{Methods}

\subsubsection{Classification of UAV RGB Orthomosaics and Calculation of CRC Data to $20 \mathrm{~m}$ Scale}

SVM is a powerful supervised classification technique and provided a superior performance compared to most other image classification methods [36,37]; therefore, it was used to classify the UAV RGB orthomosaics of the study area (Figure 1). The classification of the RGB orthomosaics 
was finished by SVM embedded in the ENVI 5.3 software. The main land cover types included soil, crop residue, tillage shadows, plastics, and burned areas. The red, green, and blue digital numbers from the RGB orthomosaic of these ground components show discrepancies, which were used in the SVM classification. The whole UAV RGB orthomosaic was clipped into 14 subsets and classified one by one. The regions of interest (ROIs) of soil, crop residue, shadows, plastics and burned areas, were collected based on the manual interpretation of the RGB image, for the image had a super-high resolution of $4.4 \mathrm{~cm}$. ROIs were created for each subset by using the ROI tool embedded in the ENVI 5.3 software. In total, we created more than 1000 ROIs for the four classes. Each ROI covered about 20-30 pixels in average. The separability between the ROIs of crop residue and the other classes was guaranteed higher than 1.8. Of these, 70\% were used for training and the remaining were used for evaluation. The classification was evaluated according to overall, user's, and producer's accuracies.
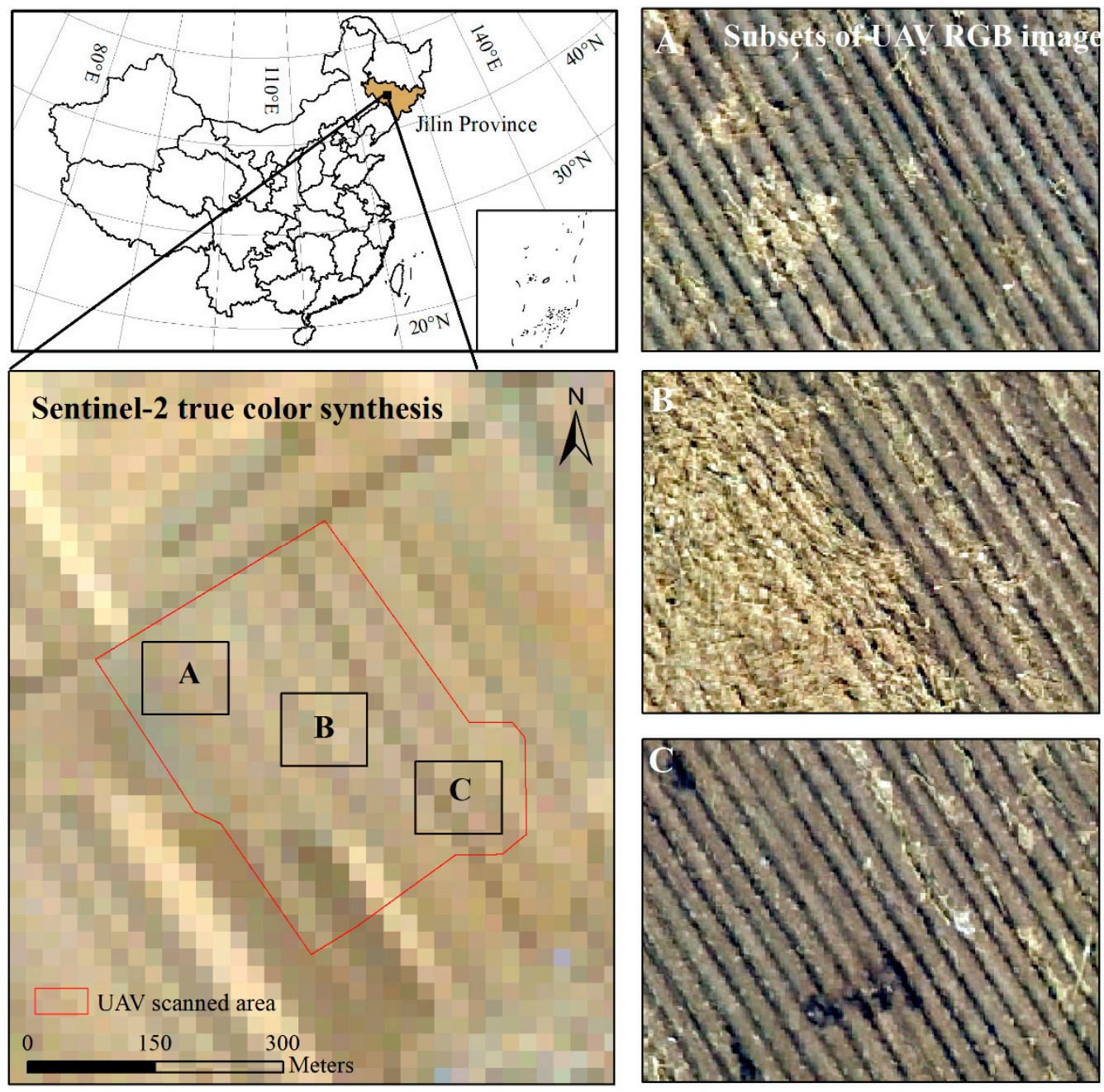

Figure 1. The location of the study area. Imagery on the left: Sentinel-2 true color synthesis of bands 2, 3, and 4. Imageries on the right: examples of unmanned aerial vehicle (UAV) images for sites (A-C).

The fractional cover of crop residue is calculated as the number of pixels in the crop residue class divided by the total number of pixels in a given grid cell (or as the mean value of the grid cell from a binary raster). The grid cell in this study is $20 \mathrm{~m}$, i.e., the spatial resolution of Sentinel-2 MSI imagery. We created precisely aligned polygon grids for Sentinel-2 $20 \mathrm{~m}$ data using the create fishnet tool in ArcMap v.10.5. The CRC was calculated to grid cells by using the zonal statistics tool in ArcMap v.10.5 based on the classification of UAV image. The study area covered $24320-\mathrm{m}$ pixels. The UAV-derived CRC (hereafter UAV-CRC, $n=243$ ) was used for the development of the CRC estimation model. 


\subsubsection{Empirical Regressions}

Empirical regression has long been the most popular method in optical remote sensing analysis [25]. In this study, we selected 10 CRIs to conduct univariate regression analysis with UAV-CRC, respectively. Eight of the ten indices were based on normalized difference format like the NDTI. Simple tillage index (STI) was the ratio of near infrared (NIR) band and SWIR band [2,19]. The deaf fuel index (DFI) was developed based on four bands of 1, 2, 6, and 7 in the Moderate Resolution Imaging Spectroradiometer (MODIS) and showed good potential in estimating steppe residue cover [38].

In addition to the ten published indices, we constructed three index formulations to investigate the potential of improving retrieval accuracy of CRC based on Sentinel-2 bands. The formulae of CAI and lignin Cellulose absorption index (LCA) [39] were adopted to calculate three-band indices (Equations (1) and (2)). These two indices have shown good performance in estimating CRC [39]. Using the NDRI formula as a basis, we constructed the third formulation by substituting one of the two bands in the denominator of NDRI with a third band (Equation (3)). These three formulations were composed of three bands. We named them three-band index 1 (3BI1), 3BI2, and 3BI3

$$
\begin{gathered}
3 \mathrm{BI} 1_{\mathrm{a}, \mathrm{b}, \mathrm{c}}=100 \times\left(0.5 \times\left(\rho_{a}+\rho_{b}\right)-\rho_{c}\right) \\
3 \mathrm{BI} 2_{\mathrm{a}, \mathrm{b}, \mathrm{c}}=\left(\rho_{a}-\rho_{b}\right) /\left(\rho_{a}-\rho_{c}\right) \\
3 \mathrm{BI} 3_{\mathrm{a}, \mathrm{b}, \mathrm{c}}=\left(\rho_{a}-\rho_{b}\right) /\left(\rho_{a}+\rho_{c}\right)
\end{gathered}
$$

\begin{tabular}{|c|c|c|c|}
\hline Vegetation Index & Abbreviation & Formula & Reference \\
\hline Normalized Difference Index 7 & NDI7 & $(\mathrm{B} 8 \mathrm{a}-\mathrm{B} 12) /(\mathrm{B} 8 \mathrm{a}+\mathrm{B} 12)$ & [20] \\
\hline Normalized Difference Residue Index & NDRI & $(\mathrm{B} 4-\mathrm{B} 12) /(\mathrm{B} 4+\mathrm{B} 12)$ & [22] \\
\hline Normalized difference senescent vegetation index & NDSVI & $(\mathrm{B} 4-\mathrm{B} 11) /(\mathrm{B} 4+\mathrm{B} 11)$ & [21] \\
\hline shortwave green normalized difference index & SGNDI & $(\mathrm{B} 3-\mathrm{B} 12) /(\mathrm{B} 3+\mathrm{B} 12)$ & [13] \\
\hline Simulated crop residue cover & MCRC & $(\mathrm{B} 11-\mathrm{B} 3) /(\mathrm{B} 11+\mathrm{B} 3)$ & [17] \\
\hline Simple tillage index & STI & $\mathrm{B} 8 \mathrm{a} / \mathrm{B} 12$ & [19] \\
\hline Dead fuel index & DFI & $100 \times(1-\mathrm{B} 12 / \mathrm{B} 11) \times(\mathrm{B} 4 / \mathrm{B} 8 \mathrm{a})$ & [38] \\
\hline Simulated cellulose absorption index & 3BI1 & $100 \times(0.5 \times(\mathrm{B} 2+\mathrm{B} 12)-\mathrm{B} 4)$ & This paper \\
\hline Simulated lignin Cellulose Absorption Index & $3 \mathrm{BI} 2$ & $(\mathrm{~B} 2-\mathrm{B} 4) /(\mathrm{B} 2-\mathrm{B} 12)$ & This paper \\
\hline
\end{tabular}

where $\rho$ represents band of Sentinel-2.

Table 1. Spectral indices used for estimating crop residue cover (CRC).

Note: Sentinel-2 MSI B2 $(490 \mathrm{~nm})$, B3 $(560 \mathrm{~nm})$, B4 $(665 \mathrm{~nm})$, B8a $(865 \mathrm{~nm})$, B11 $(1610 \mathrm{~nm})$, and B12 $(2190 \mathrm{~nm})$ represent multispectral bands of blue, green, red, near-infrared, SWIR 1 and SWIR 2.

In order to select a best-performing index, all possible band combinations were correlated with the UAV-measured CRC according to the three formulae. The band combination with the highest coefficient of determination $\left(R^{2}\right)$ for each formula was selected as the optimal index. The optimal indices output from the three formulae are listed in Table 1. All the three indices used Sentinel-MSI band 2, 4, and 12. The 3BI3 also used band 11.

The performance of the nine bands of Sentinel-2 with a spatial resolution of $20 \mathrm{~m}$ on CRC estimation were also investigated. Furthermore, we extracted mean textural feature, which was reported as being correlated to CRC [35], by using a gray level co-occurrence matrix from Sentinel-2 10 and $20 \mathrm{~m}$ imagery, respectively. The mean textural features of each Sentinel-2 $10 \mathrm{~m}$ band- B2, B3, B4, and B8- were aggregated to $20 \mathrm{~m}$ grid cells to match the pixel size of Sentinel-2 $20 \mathrm{~m}$ image using the zonal statistics tool in Arcmap v.10. A pre-primary analysis showed that the aggregated $20 \mathrm{~m}$ mean textures appear more correlated with CRC in comparison to the mean textural features, directly derived from $20 \mathrm{~m}$ MSI imagery. Therefore, the aggregated mean textural features of band 2, 3, 4, and 8 were employed. 
Each of the bands, indices, and textural features was used as a single explanatory variable for retrieval of CRC. Preprimary analysis showed that these predictors had linear relationships with the UAV-CRC. Therefore, we restricted the fitting method to ordinary least-squares linear regression.

\subsubsection{Machine Learning Methods}

In contrast to empirical regression, $\mathrm{ML}$ algorithms are robust to noisy data and can approximate multivariate non-linear relationships. The PLSR is a linear non-parametric regression model. ANN, GRP, SVR, and RF, also referred to as non-linear non-parametric models, apply non-linear transformations. We grouped input predictors into four categories, i.e., nine MSI bands, 13 CRIs, a combination of MSI bands and the four aggregated textural features, and a combination of 13 CRIs and those textural features. The output parameter was the corresponding UAV-CRC measurements.

The PLSR decomposes both the dependent and independent variables into a number of principal components, and can accommodate highly correlated variables and over-fitting. It is a bilinear calibration method that utilizes data compression by reducing a large number of measured collinear variables to a few non-correlated factors [41].

ANNs have been widely used for estimating terrestrial variables from remote sensing data [25,42]. We used a back-propagation ANN that consisted of input, hidden and output layers. The activation functions in the hidden and output nodes were set to "sigmoid" and linear, respectively. The Levenberg-Marquardt minimization algorithm was used to calibrate the synaptic coefficients [43]. In order to optimize the structural parameters of ANN for the network, we varied both the momentum coefficient and learning rate from 0.1 to 1.0 , at a step of 0.05 . The number of nodes in the hidden layer was varied from three to seven. The mean squared error with a value of $1 \mathrm{e}-6$ was used as the performance threshold. The optimal network was selected in terms of the absolutely average error between the validation data and predictions.

The SVR model by Vapnik et al. [44], is a popular tool for solving non-linear problems. In the SVR procedure, the n-dimensional input variables are conveyed into a new feature space with higher dimensions using kernel functions, and consequently, the optimal separating hyperplanes are developed [45]. In this study, a Gaussian kernel was applied as the kernel function. Other kernel types-linear, quadratic, and cubic - were tested, but the Gaussian kernel method was found to be the most reliable. The remaining parameters, including the box constraint mode, kernel scale, and the epsilon mode, were optimized.

GPR is another popular kernel-based ML method for the non-linear regression problem [46]. In the process of training data, GPR first elicited a prior GPR to constrain the possible forms of the unknown function and then updates this prior in the light of training samples to generate a posterior GPR as the final functional model [47]. We used an exponential kernel and a constant basic function. The parameter sigma was optimized.

The RF is an ensemble-learning algorithm that combines a large set of decision trees to improve prediction accuracy [48]. The RF presents several advantages: it is not sensitive to noise or over-fitting, and it can handle thousands of input variables without variable deletion and runs efficiently [28,49]. In order to implement the RF, two parameters needed to be set up: the number of trees and the number of features. In this study, we applied grid searching and varied the number of trees from 50 to 500 at a step size of 20. The number of features is all the input variables. The optimal network was selected in terms of the absolutely average error between the validation data and predictions. A diagram of the workflow is provided in Figure 2. The hyperparameters of ANN, GPR, SVR, and RF are represented in the Supplementary Materials (Tables S1-S4).

\subsection{Model Calibration and Validation}

The performances of the above estimation algorithms were evaluated using $R^{2}$ and root mean square error (RMSE) with respect to the UAV measured CRC. In order to avoid over-fitting of the ML algorithms and dependence on a single random partitioning of the datasets, as well as guarantee that 
all samples were used for both training and validation, we used a repeated 10-fold cross-validation procedure in the training process and evaluated the performance of each approach, e.g., as reported in other studies $[23,28,50]$. Samples were randomly split into 10 equal-sized sub-datasets, and they were trained and tested 10 times. For each time, nine sub-datasets were used iteratively for calibration and the remaining sub-dataset for validation. The cross-validation process was then repeated 10 times. By repeating the training procedure 10 times, all observations are used for both calibration and validation, with each observation used for validation only once in one of 10 iterations. The $R^{2}$ cv and $\mathrm{RMSE}_{\mathrm{cv}}$ of each algorithms represented here are the average of 10 iterations. The $R^{2}{ }_{\mathrm{cv}}$ and $\mathrm{RMSE}_{\mathrm{cv}}$ of each fold for each algorithms is supplied in the Supplementary Materials (Tables S5-S11).

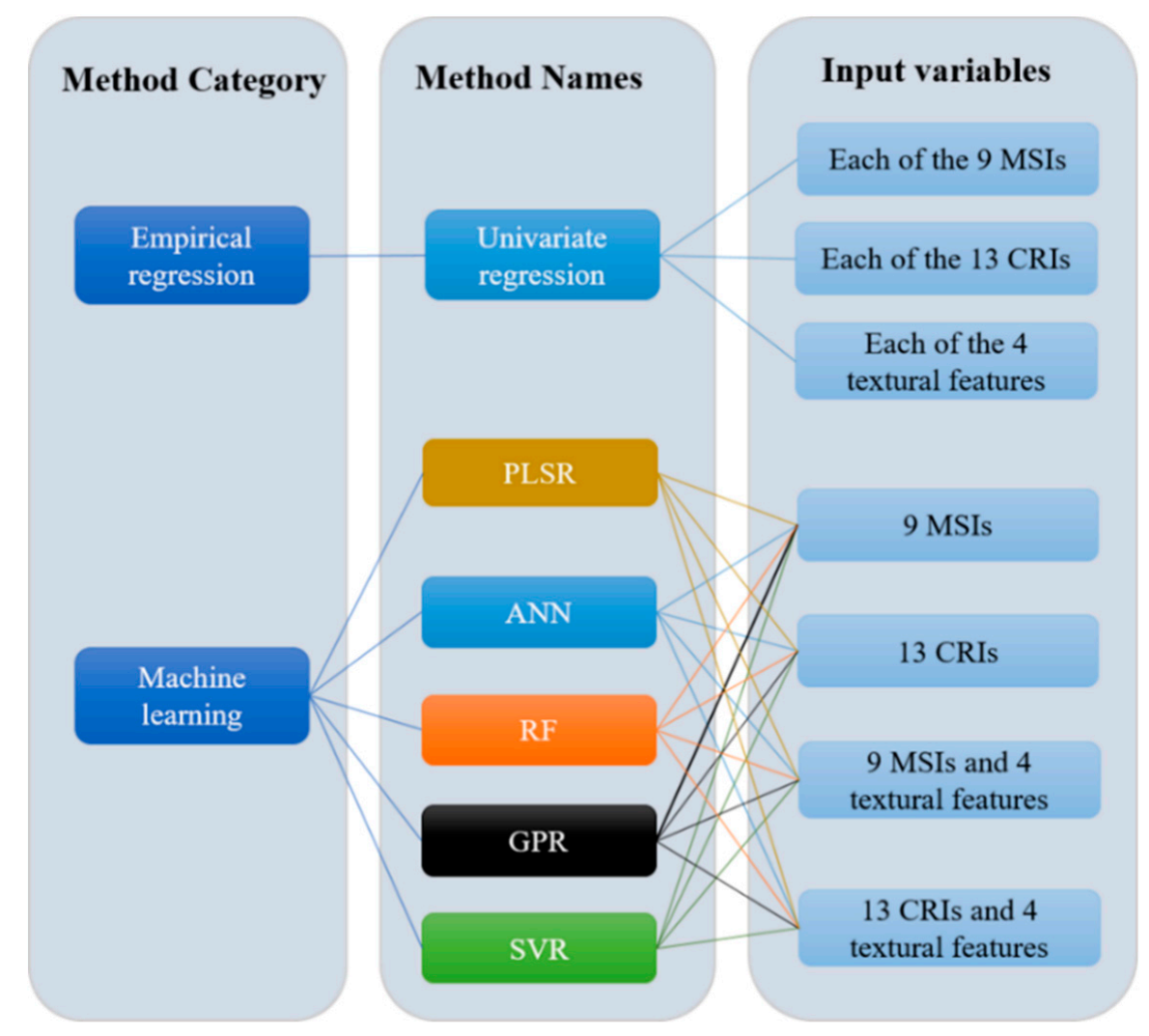

Figure 2. A flow chart of methods used for estimating CRC from Sentinel-2 imagery.

\section{Results}

\subsection{Crop Residue Classfication Based on UAV Images}

Figure 3 shows examples of the SVM classification results. The classification had a high accuracy (Table 2). The overall accuracy was about $98.06 \%$. The producer's accuracy for the crop residue class was $97.47 \%$ and the user's accuracy was $98.86 \%$. The corresponding values for the soil class were $98.73 \%$ and $97.20 \%$.

Table 2. Confusion matrix for the SVM classification with validating data (number of pixels $=5900$ ).

\begin{tabular}{ccc}
\hline \multirow{2}{*}{ Classified } & \multicolumn{2}{c}{ Reference } \\
\cline { 2 - 3 } & Crop Residue & Soil \\
\hline Crop residue & 3047 & 35 \\
Soil & 79 & 2739 \\
\hline
\end{tabular}




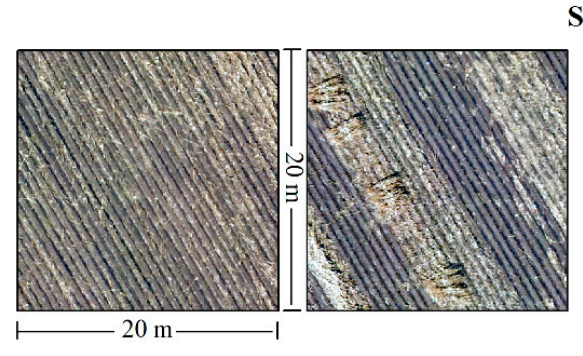

Subsets of UAV RGB images
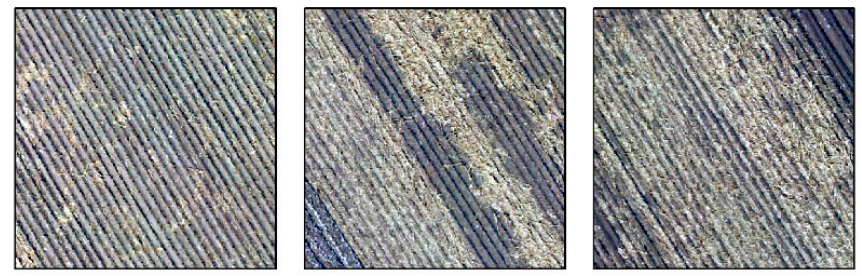

SVM Classifications
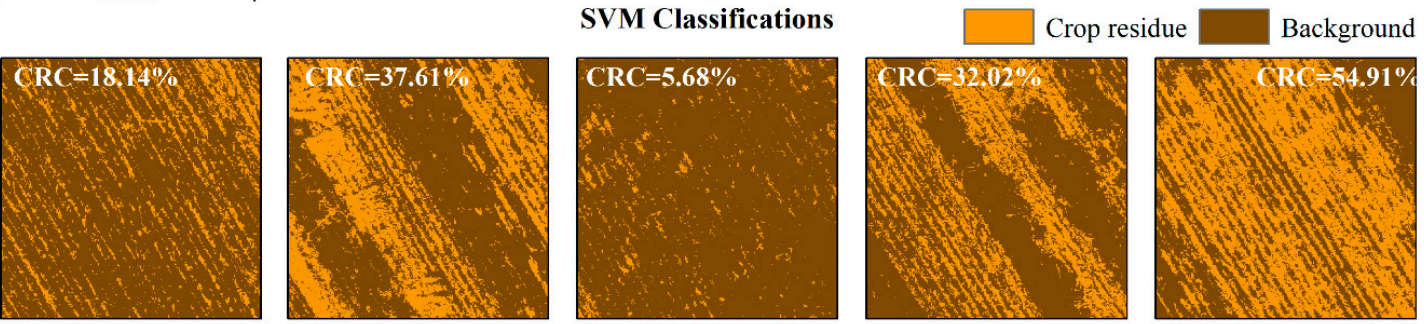

Figure 3. Examples of support vector machine (SVM) classification results. The upper figures are subsets of UAV mosaic and the lower figures are corresponding classifications.

\subsection{Empirical Regression Analysis Using Sentinel-2 Bands, Indices, and Textural Features for CRC Estimation}

Figure 4 highlights the correlation matrices of all the predictor variables derived from Sentinel-2 and the UAV measured CRC. All bands were highly correlated with CRC with Pearson's correlation coefficients between 0.53 and 0.75 , except band 12. Band 4 had the strongest relationship $(R=0.75)$ with CRC in these nine bands. Most of the 10 published indices showed high positive correlations with the exception of MCRC. NDRI had a correlation of 0.77 with CRC, followed by NDI7 and STI. The proposed 3BI2 was the most positively correlated with CRC $(R=0.78)$. The proposed 3BI1 was the most negatively correlated with CRC $(\mathrm{R}=-0.78)$, followed by 3BI3 and NDSVI. The mean textures extracted from bands 2, 3, 4, and 8 were also correlated with CRC. The mean texture derived from band 4 (named B4_mean) performed better than the other textures. In general, CRIs were more correlated with CRC than bands and textural features. The 26 variables showed different degrees of correlation with each other.

The performance of the above variables on estimating CRC was first analyzed by least-squares linear regression between each of variables and the UAV measured CRC. The results of the 10-fold cross-validation for the models (Table 3) revealed that the models based on MSI bands presented various performances, with $R^{2}$ cv value ranging from 0.07 to 0.58 and $\mathrm{RMSE}_{\mathrm{cv}}$ ranging from $10.902 \%$ to $7.185 \%$. Among the nine bands, the best model was fitted by band 4 , which yielded the highest correlation with CRC $\left(R^{2}{ }_{\mathrm{cv}}=0.58, \mathrm{RMSE}_{\mathrm{cv}}=7.185 \%\right)$, followed by models based on band 5 and 8a. The regression relationship between band 4 and CRC is shown in Figure 5a. The most poorly performing band was band 12 , with an $R^{2}{ }_{\mathrm{cv}}$ of 0.07 and $\mathrm{RMSE}_{\mathrm{cv}}$ of $10.902 \%$. The performance order of MSI bands from the highest to lowest with respect to RMSE $\mathrm{cv}_{\mathrm{c}}$ values was B4, B5, B7, B8a, B3, B6, B2, $\mathrm{B} 11$, and B12.

The models based on published indices yielded various retrieval results, with $\mathrm{R}_{\mathrm{cv}}$ values ranging from 0.15 to 0.61 and RMSE ranging from $6.663 \%$ to $10.336 \%$ (Table 3). NDRI calculated by band 4 and band 12 performed best, with an $R^{2}$ cv of 0.61 and RMSE $_{\mathrm{cv}}$ of $6.663 \%$, followed by NDI7 and STI, which were calculated by bands $8 \mathrm{a}$ and 12 . The DFI, a combination of bands $12,11,4$, and $8 \mathrm{a}$, also presented a reliable estimate. NDTI provided an accuracy of $R^{2}{ }_{\mathrm{cv}}$ equal 0.50 and $\mathrm{RMSE}_{\mathrm{cv}}$ equal to $7.585 \%$. Other indices presented relatively poor performance, especially MCRC, which was proposed for differentiating between conventional and conservation tillage [17]. Among all the CRIs, the best retrieval accuracy was achieved by $3 \mathrm{BI} 2\left(R_{\mathrm{cv}}^{2}=0.63, \mathrm{RMSE}_{\mathrm{cv}}=6.509 \%\right)$, followed by 3BI1. These two indices, developed from the formulae of CAI and LCA, performed better than the 10 published indices. Adding a third band at the basis of NDRI, 3BI3 slightly improved the accuracy compared to NDRI. 
The three proposed indices were associated with MSI bands 4, 12, and 2. Although band 12 was not correlated to CRC, indices utilizing it performed better than those without it, such as NDI7 versus NDI5 and NDRI versus NDSVI. This is likely due to the cellulose and lignin absorption in residue near band 12 [22]. The scatter plots between CRC and the best four indices (NDRI, 3BI1, 3BI2, and 3BI3) are shown in Figure 5b-e.

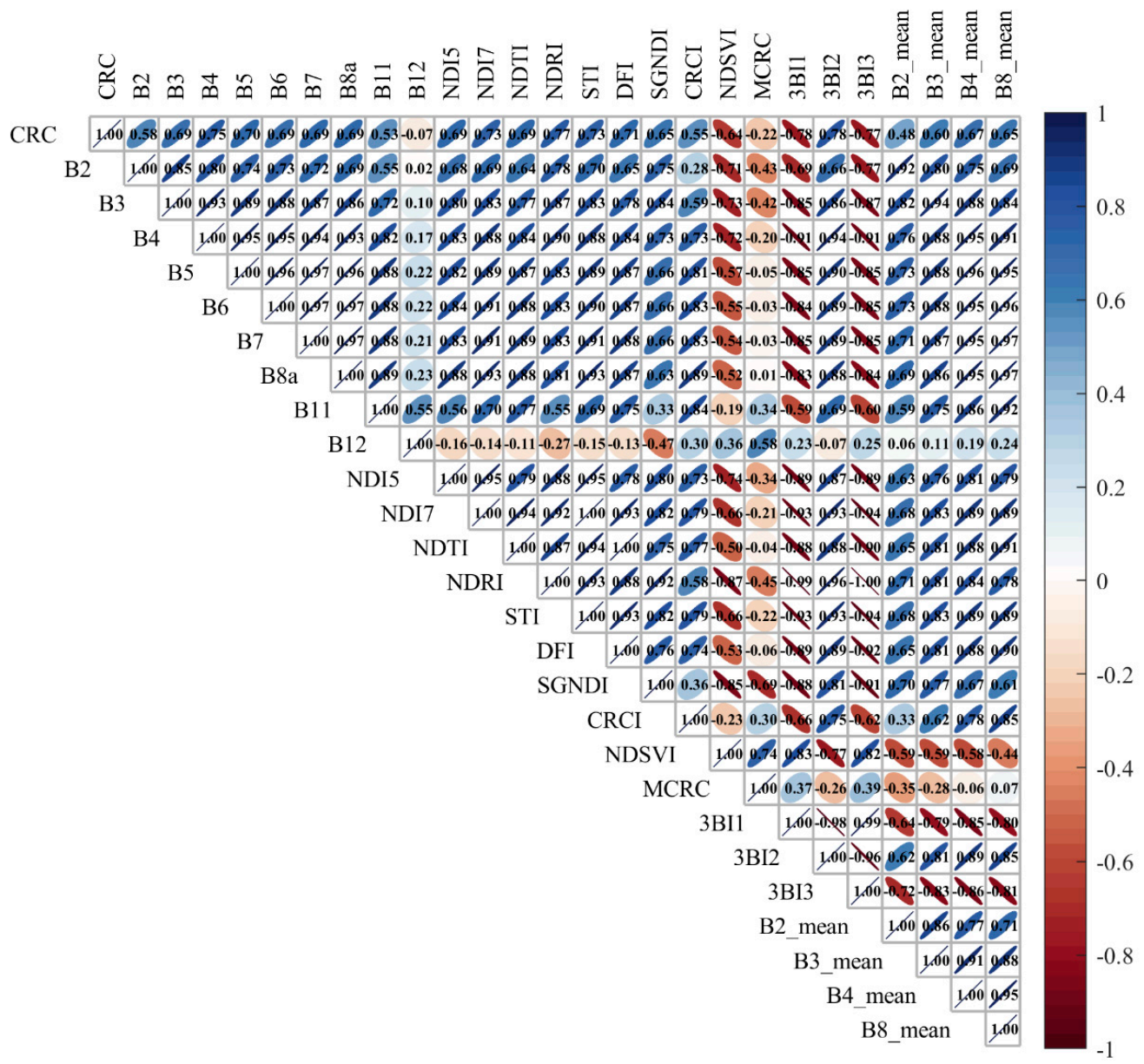

Figure 4. Correlation matrix for CRC and predictor variables including MSI bands, indices and textural features. The Sentinel-2 MSI bands are represented as B2, B3, .. , B12. The mean textural feature are represented as B2_mean, B3_mean,... , B8_mean. The length on the minor axis of the ellipse indicates the strength of the Pearson's correlation coefficient between each pair variables, while the colors of the ellipse indicate whether the correlation is positive or negative.

Table 3. Performance of MSI bands, crop residue indices (CRIs) and textural features on estimating CRC. The best-performing variables for each category are highlighted in boldface.

\begin{tabular}{cccc}
\hline Category & Predictor Variables & $\boldsymbol{R}_{\mathbf{c v}}$ & RMSE $_{\mathbf{c v}}(\mathbf{\%})$ \\
\hline MSI bands & B2 & 0.35 & 8.936 \\
& B3 & 0.48 & 7.942 \\
B4 & $\mathbf{0 . 5 8}$ & 7.185 \\
B5 & 0.49 & 7.847 \\
B6 & 0.49 & 7.943 \\
B7 & 0.46 & 7.895 \\
B8a & 0.50 & 7.903 \\
& B11 & 0.32 & 9.288 \\
& B12 & 0.07 & 10.902 \\
\hline
\end{tabular}


Table 3. Cont.

\begin{tabular}{cccc}
\hline Category & Predictor Variables & $\boldsymbol{R}_{\mathbf{c v}}$ & $\mathbf{R M S E}_{\mathbf{c v}} \mathbf{( \% )}$ \\
\hline CRIs & NDI5 & 0.51 & 7.623 \\
NDI7 & 0.57 & 7.162 \\
NDTI & 0.50 & 7.585 \\
NDRI & 0.61 & 6.663 \\
NDSVI & 0.42 & 8.107 \\
SGNDI & 0.45 & 7.902 \\
CRCI & 0.33 & 8.804 \\
MCRC & 0.15 & 10.336 \\
& STI & 0.57 & 7.178 \\
DFI & 0.52 & 7.441 \\
& 3BI1 & $\mathbf{0 . 6 1}$ & $\mathbf{6 . 5 9 8}$ \\
& 3BI2 & $\mathbf{0 . 6 3}$ & $\mathbf{6 . 5 0 9}$ \\
& 3BI3 & $\mathbf{0 . 6 1}$ & $\mathbf{6 . 6 3 0}$ \\
\hline Textural features & B2_mean & 0.28 & 9.466 \\
& B3_mean & 0.40 & 8.652 \\
& B4_mean & $\mathbf{0 . 4 9}$ & $\mathbf{8 . 0 7 1}$ \\
& B8_mean & 0.47 & 8.220 \\
\hline
\end{tabular}
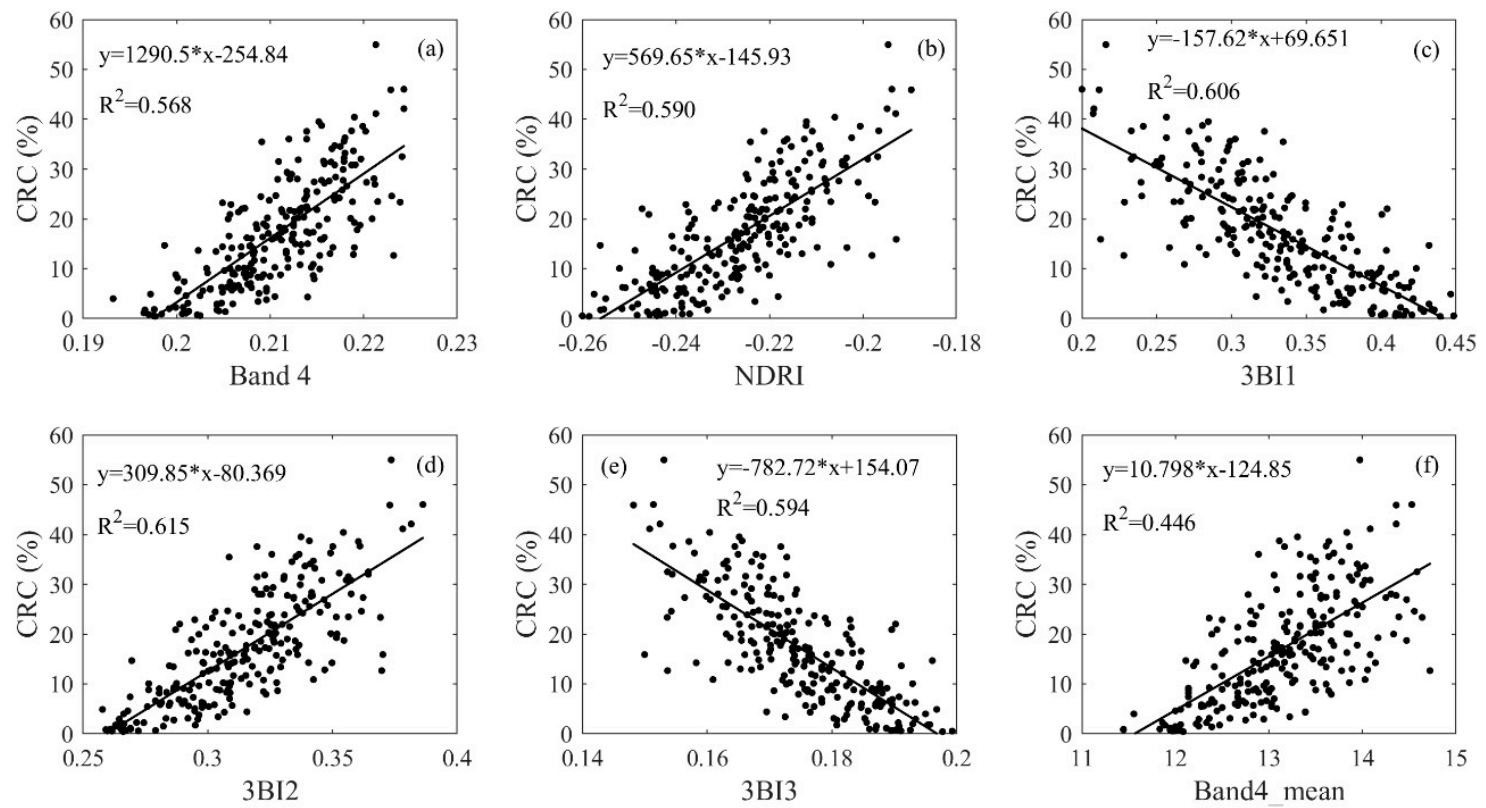

Figure 5. Regression relationships between CRC and the best predictors in each category ((a): Band 4, (b): NDRI, (c): 3BI1, (d): 3BI2, (e): 3BI3, (f): Band4_mean) (Table 3).

Of the four aggregated mean textural features investigated, the mean feature derived from MSI band 4 led to the most accurate estimates of $C R C\left(R^{2}{ }_{c v}=0.49, R_{\text {MSE }}=8.071 \%\right)$, followed by B8_mean and B3_mean. B2_mean performed poorly. The regressed relationship between B4_mean and CRC is shown in Figure 5f. Models based on textural features presented higher RMSE values than those based on bands and indices. Generally, CRIs performed best.

\subsection{Machine Learning Approaches for CRC Estimation.}

\subsubsection{Performance of PLSR on Estimating CRC}

PLSR, an extension of multiple linear regression technique, is particularly useful in the case of predicting a set of dependent variables from a large set of independent variables. The above MSI bands, CRIs, and textural features were correlated with CRC in different degrees. Combinations of these 
features via PLSR were expected to offer advantages over univariate regression, including enhancing the sensitivity to crop residue, reducing the influence of background spectral noise, and providing structural information via textural features. Therefore, we applied four groups of variables, i.e., MSI nine bands, 13 CRIs, a combination of nine bands and four mean textural features, and a combination of 13 CRIs and those textural features, to estimate CRC via PLSR (Figure 2).

Table 4 presents a comparative analysis of the performance level of PLSR models based on the four groups of input variables. The PLSR model based on the nine MSI bands generated better results than any univariate models based on the MSI bands in terms of $R^{2}{ }_{\mathrm{cv}}$ and $\mathrm{RMSE}_{\mathrm{cv}}$. Similarly, the PLSR model based on the 13 CRIs yielded a higher accuracy of estimating CRC compared to NDRI and NDTI. In addition, the PLSR combining nine bands and four textural features contributed to a lower RMSE $\mathrm{Ev}_{\mathrm{c}}$ compared to that based on nine bands. The retrieval accuracy of PLSR was further improved by using 13 CRIs and four textural features as input features $\left(R^{2}{ }_{\mathrm{cv}}=0.66, \mathrm{RMSE}_{\mathrm{cv}}=6.427 \%\right)$. The addition of textural features enriched the effective information for CRC estimation. The scatter plot of measured CRC values versus estimated value by the best PLSR model is shown in Figure 6. It is noted that samples with CRC measurements below a value of $20 \%$ were systematically overestimated and those higher than $20 \%$ were underestimated.

Table 4. The performances of partial least squares regression (PLSR) associated with four groups of input predictors on CRC estimation. The best-performing input variable combination is highlighted in boldface.

\begin{tabular}{ccc}
\hline Input Variables & $\boldsymbol{R}_{\mathbf{c v}}^{\mathbf{2}}$ & $\mathbf{R M S E}_{\mathbf{c v}} \mathbf{( \% )}$ \\
\hline 9 Bands & 0.59 & 6.822 \\
13 CRIs & 0.65 & 6.576 \\
9 Bands and 4 mean textural features & 0.63 & 6.746 \\
13 CRIs and 4 mean textural features & $\mathbf{0 . 6 6}$ & $\mathbf{6 . 4 2 7}$ \\
\hline
\end{tabular}

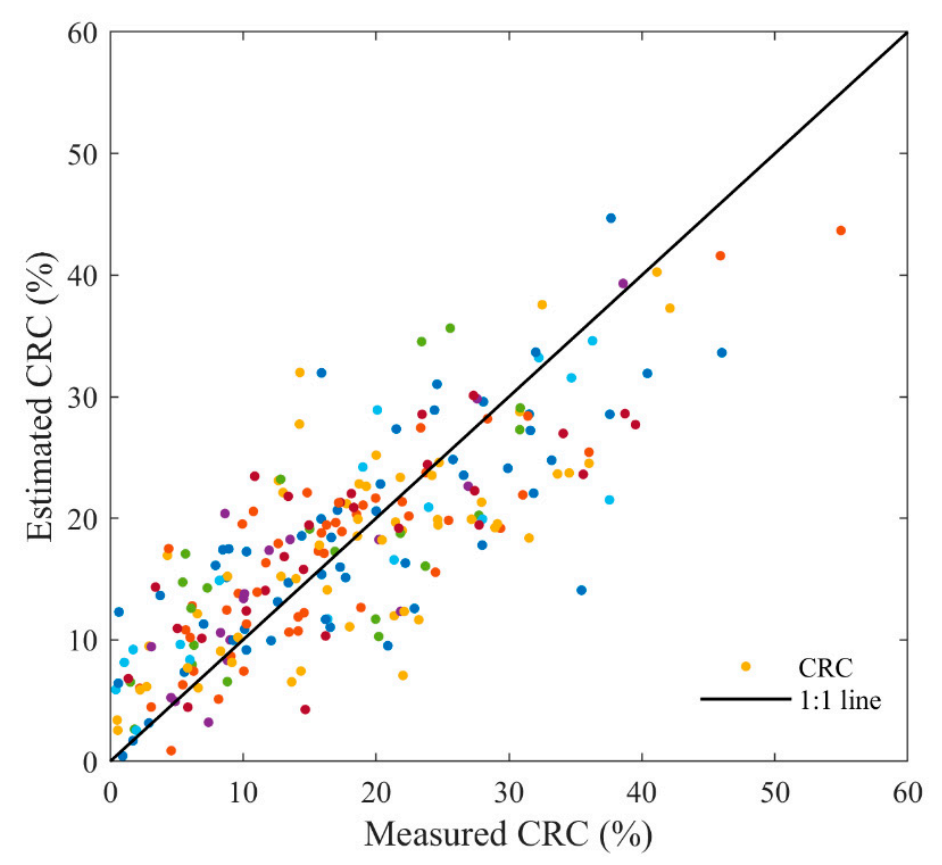

Figure 6. Measured vs. estimated CRC values along the 1:1 line of the best PLSR model (see Table 4). The different colors indicate the 10-fold subsets.

\subsubsection{Performance of ANN, GPR, SVR, and RF on Estimating CRC}

Table 5 shows the cross-validation results of the four non-linear non-parametric algorithms-ANN, GPR, SVR, and RF- with four groups of input predictors. Generally, every approach presented reliable 
estimates with acceptable $R^{2}{ }_{\mathrm{cv}}$ and $\mathrm{RMSE}_{\mathrm{cv}}$ values. The ANN models were highly correlated with the UAV-CRC, but with higher RMSE values than the results derived from the RF. These four ML algorithms based on MSI bands outperformed the large majority of univariate regressions while not performing as well as 3BI2. Further analysis was executed by considering CRIs as input variables as well as a combination of bands and textural features, and a combination of CRIs and textural features (Figure 2).

Table 5. Performance of artificial neural network (ANN), Gaussian process regression (GPR), support vector regression (SVR), and random forest (RF) associated with four groups of input predictors on CRC estimation. The best-performing input variable combination for each algorithm is highlighted in boldface.

\begin{tabular}{cccc}
\hline Algorithms & Input Variables & $\boldsymbol{R}_{\mathbf{c v}}{ }^{2}$ & $\mathbf{R M S E}_{\mathbf{c v}} \mathbf{( \% )}$ \\
\hline ANN & 9 Bands & 0.62 & 8.324 \\
& 13 CRIs & 0.59 & 7.633 \\
& 9 Bands and 4 mean textural features & $\mathbf{0 . 6 9}$ & $\mathbf{7 . 2 7 3}$ \\
& 13 CRIs and 4 mean textural features & 0.63 & 7.486 \\
\hline RF & 9 Bands & 0.59 & 6.695 \\
& 13 CRIs & 0.60 & 6.499 \\
& 9 Bands and 4 mean textural features & $\mathbf{0 . 6 1}$ & $\mathbf{6 . 4 1 5}$ \\
& 13 CRIs and 4 mean textural features & 0.59 & 6.470 \\
\hline GPR & 9 Bands & 0.64 & 6.593 \\
& 13 CRIs & 0.64 & 6.579 \\
& 9 Bands and 4 mean textural features & 0.66 & 6.382 \\
& 13 CRIs and 4 mean textural features & $\mathbf{0 . 6 6}$ & $\mathbf{6 . 3 5 2}$ \\
\hline SVR & 9 Bands & 0.62 & 6.719 \\
& 13 CRIs & 0.63 & 6.692 \\
& 9 Bands and 4 mean textural features & $\mathbf{0 . 6 7}$ & $\mathbf{6 . 3 4 3}$ \\
& 13 CRIs and 4 mean textural features & 0.66 & 6.393 \\
\hline
\end{tabular}

Compared to utilizing MSI bands as input predictors, the use of the other three groups of input variables improved the performance of the four non-linear ML approaches (Table 5). In terms of ANN, the retrieval accuracy was significantly improved by replacing bands with either CRIs, a mixture of bands and textural features, or a combination of CRIs and textures. The highest accuracy $\left(R^{2} \mathrm{cv}=0.69\right.$, $\mathrm{RMSE}_{\mathrm{Cv}}=7.273 \%$ ) was obtained by using bands and textures as input predictors. Similarly, the retrieval accuracy obtained by employing RF with either CRIs, a mixture of bands and textures, or a mixture of CRIs and textures, was also improved compared to only using bands as input predictors. The best RF performance was found with bands and textures as input variables $\left(R_{\mathrm{cv}}^{2}=0.61, \mathrm{RMSE}_{\mathrm{cv}}=6.415 \%\right)$. Although using CRIs to replace bands in GPR and SVR models obtained little improvement, these two algorithms associated with the latter two groups of variables obviously improved their performance. For GPR model, the lowest RMSE $\mathrm{cv}_{\mathrm{cv}}$ and the highest $R_{\mathrm{cv}}^{2}$ were obtained by combining CRIs and textures $\left(R_{\mathrm{cv}}^{2}=0.66, \mathrm{RMSE}_{\mathrm{cv}}=6.352 \%\right)$. The best-performing model was SVR associated with bands and textures $\left(R^{2}{ }_{\mathrm{cv}}=0.67, \mathrm{RMSE}_{\mathrm{cv}}=6.343 \%\right)$. The results of these four ML models also demonstrated that adding texture information improved retrieval accuracy.

When comparing the PLSR and ML models, the best RF, GPR, and SVR performed better than the best PLSR model. A higher performance for these three non-linear ML models implies that the relationship between CRC and input variables may be non-linear. PLSR cannot cope with complex nonlinear relations [23]. The GPR with CRIs and textural features as input variables and the SVR with an input of bands and textural features are robust for CRC estimation. 


\subsubsection{Optimization of the GPR Model}

As noted earlier, one of the advantages of GPR is its ability to provide insight into the relevance of input predictors. The relevance can be interpreted by $\sigma$, which is a parameter of the covariance function of GPR: $\mathrm{K}\left(x_{i}, x_{j}\right)=\exp \left(-\frac{\left\|x_{i}, x_{j}\right\|^{2}}{2 \sigma^{2}}\right)$. High $\sigma$ values mean that relations largely extend along that predictor; hence, the lower the $\sigma$, the more relevant the predictor [51]. We calculated $\sigma$ for each groups of input predictors and illustrated it in Figure 7. The plot of $\sigma$ for the nine bands (Figure 7a) shows that the most relevant bands were encountered in the red (B4), green (B3), and the second SWIR band (B12). Indices associated with these three bands have proven powerful in estimating CRC (Table 3). Calculation of $\sigma$ values for the 13 indices (Figure 7b) showed that 3BI1, NDSVI, SGNDI, and NDRI were more relevant to CRC than other CRIs. Those indices were related to B3, B4, and B12. When texture information and bands were inputted into GPR algorithm together, B4 and B3_mean were more relevant to CRC than other variables (Figure 7c). B2_mean was not as relevant to CRC as the other three textural features. As for the combination of CRIs and textures as input predictors, NDI5, NDSVI, 3BI1 and 3BI2 presented lower $\sigma$ than other indices (Figure 7d). The mean textures of B3, B4, and $\mathrm{B} 8$ were also relevant to CRC.

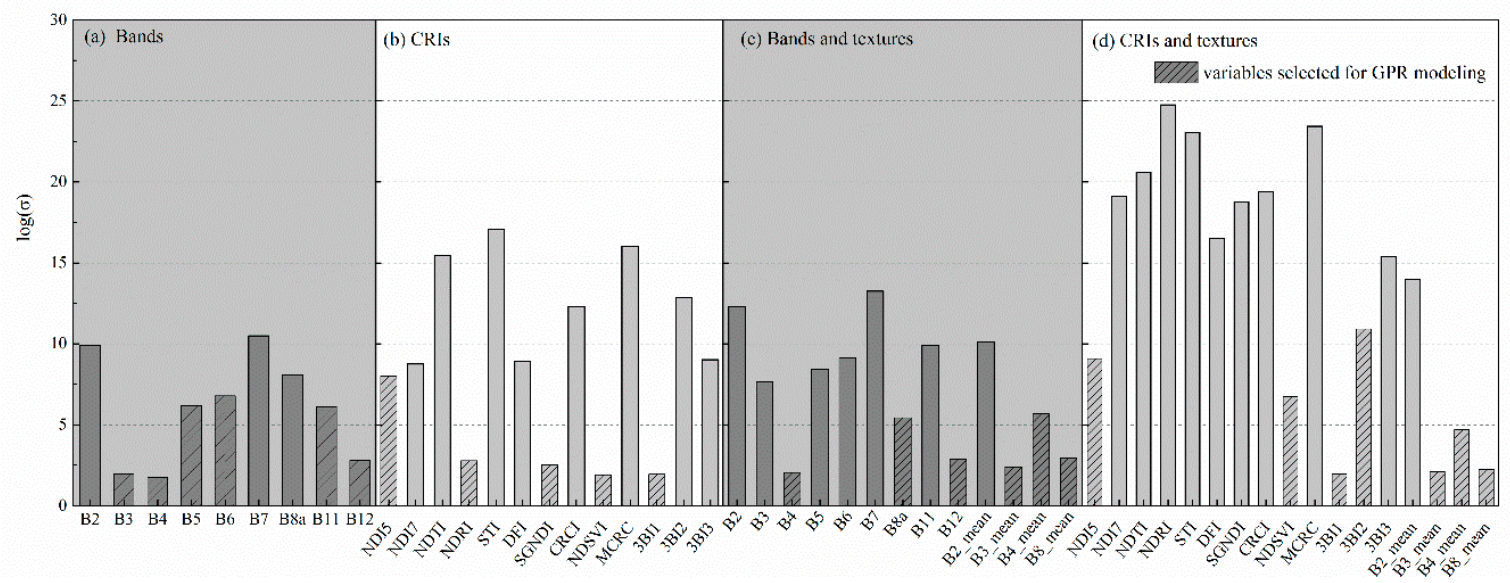

Figure 7. The logarithmic sigmas of GPR models with different groups of input predictors ((a): MSI bands, (b): CRIs, (c): Bands and textures, (d): CRIs and textures).

We explored the possibility of optimizing the GPR model by excluding the least important predictors according to the relevance of each variable to CRC. After the model evaluation using the entire set of input variables, we used a stepwise elimination method to identify the optimal input combination in such a way to reduce the number of input variables, beginning at the variable with the highest $\sigma$. We ended up with the combination that provided the lowest RMSE. The slash-filled bars in Figure 7 were final input variables for each group. Table 6 lists the accuracies retrieved by GPR models with optimal input variables. Compared to Table 5, the accuracy of each GPR algorithm was improved by selecting input variables according to $\sigma$ value. Compared to the original nine bands as input variables, the RMSE $\mathrm{cv}_{\mathrm{cv}}$ was improved from $6.593 \%$ to $6.4 \%$ by using B3, B4, B5, B6, B11, and B12 as input predictors. In terms of the GPR model based on CRIs, the 13 CRIs were reduced to NDI5, NDRI, SGNDI, NDSVI, and 3BI1 with a retrieval accuracy of $R^{2}{ }_{\mathrm{cv}}$ equal to 0.68 and RMSE $\mathrm{cv}_{\mathrm{cv}}$ equal to $6.445 \%$. The combination of NDI5, NDSVI, 3BI1, and mean textures of B3, B4, and B8 also improved GPR retrieval accuracy. The optimal variables among bands and textural features were B4, B8a, and B12, and mean textures of B3, B4, and B8. The GPR associated with these variables outperformed other GPRs, enhancing $R^{2}$ cv from 0.66 to 0.69 and $\mathrm{RMSE}_{\mathrm{cv}}$ from $6.382 \%$ to $6.149 \%$. The scatter plots between estimated CRC and measured CRC demonstrate that the CRC estimated lies close to the 1:1 line (Figure 8). Compared to the estimation results by PLSR (Figure 6), overestimation and underestimation were improved. The above results indicated that this is an effective way to identity optimal input 
variables by using $\sigma$ values. The performance of GPR model was further improved by using bands and mean textural features information.

Table 6. Performance of the optimized GPR models. The best-performing input variable combination for GPR is highlighted in boldface.

\begin{tabular}{cccc}
\hline Algorithm & Optimized Input Predictors & $\boldsymbol{R}_{\mathbf{c v}}$ & $\mathbf{R M S E}_{\mathbf{c v}}(\mathbf{\%})$ \\
\hline GPR & B3, B4, B5, B6, B11, B12 & 0.66 & 6.400 \\
& NDI5, NDRI, SGNDI, NDSVI, 3BI1 & 0.68 & 6.445 \\
& B4, B8a, B12, B3_mean, B4_mean, B8_mean & $\mathbf{0 . 6 9}$ & $\mathbf{6 . 1 4 9}$ \\
& NDI5, NDSVI, 3BI1, 3BI2, B3_mean, B4_mean, B8_mean & 0.67 & 6.325 \\
\hline
\end{tabular}

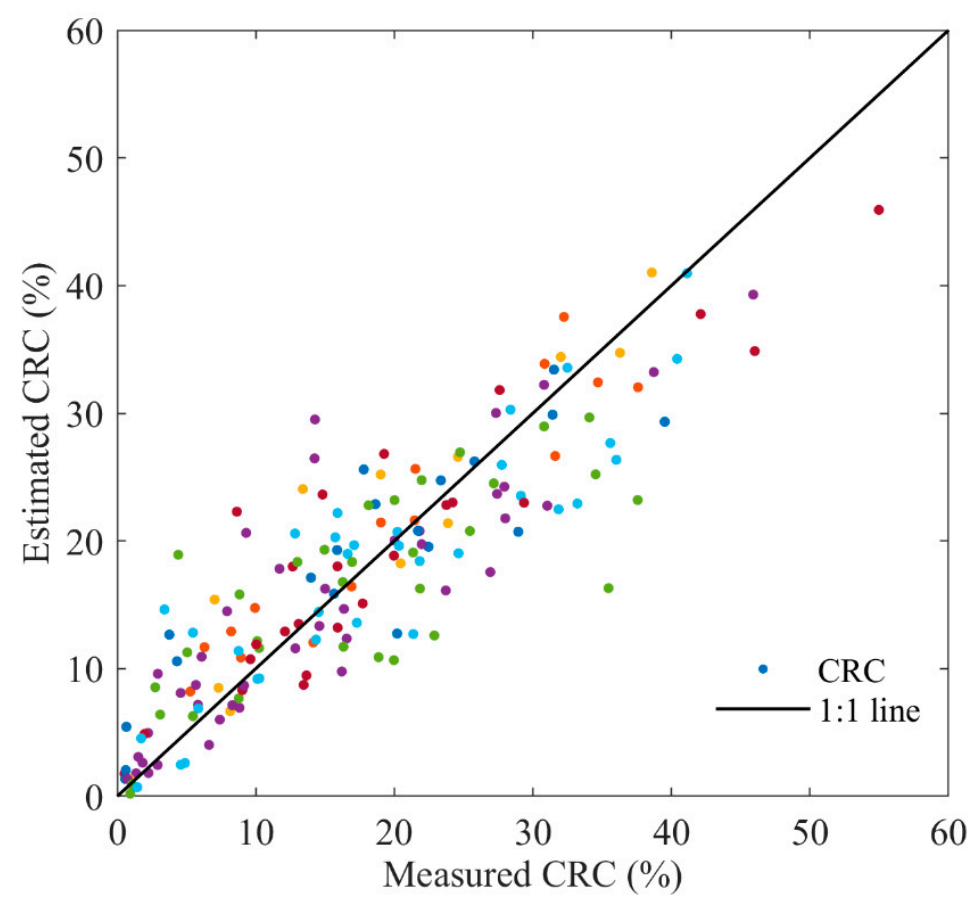

Figure 8. Measured vs. estimated CRC values along the 1:1 line of the best performing GPR mode (see Table 6). The different colors indicated the 10-fold subsets.

\section{Discussion}

In this study, we investigated empirical regression and ML methods for the purpose of estimating CRC from Sentinel-2 MSI data. Regression analysis using established CRIs and proposed CRIs based on Sentinel-2 MSI data were explored, together with the applications of PLSR and four non-linear non-parametric ML approaches (ANN, SVR, GPR, and RF), using (1) nine MSI bands, (2) 13 CRIs, (3) nine bands and four mean textural features, and (4) 13 CRIs and those textural features as predictor variables, respectively. Overall, the results demonstrated that PLSR, SVR, GPR, and RF provided higher retrieval accuracy when textural features and CRIs were used as input predictors. The GPR model performance was further improved by using optimized input predictors. GPR is recognized as a promising method for estimating CRC. A further discussion of the elements of this study is presented below.

\subsection{Empirical Regression Analysis for CRC Estimation}

Univariate regressions examining the relationship between individual bands, CRIs, and mean textural features and CRC were conducted. Most of the bands, CRIs, and textural features were correlated to CRC. The best-performing index among the published CRIs was NDRI with an accuracy of $R^{2}{ }_{\mathrm{cv}}$ of0.61 and RMSE $\mathrm{cv}_{\mathrm{cv}}$ of $6.663 \%$. The proposed index 3BI2, constructed with band 2, 4, and 
12 , obtained the best performance with $R^{2}{ }_{\mathrm{cv}}=0.63$ and $\mathrm{RMSE}_{\mathrm{cv}}=6.509 \%$, followed by $3 \mathrm{BI} 1$ and 3BI3. These three-band indices performed better than two-band indices listed in Table 1. Our results confirmed that, as noted by Verrelst et al. [23], when multiple bands are available there is no reason to limit the estimation to two-band indices. Notwithstanding the promising results, we acknowledge that further comprehensive work is needed to evaluate the reliability of the three proposed indices to estimate CRC in the context of different residue types under various circumstances, such as residue decomposition stage, residue and soil moisture content, soil brightness, and the presence of green vegetation $[7,18]$.

\subsection{Machine Learning Approaches for CRC Estimation}

In this study, five ML techniques (PLSR, ANN, SVR, GPR, and RF) were evaluated and the importance of input variables for these approaches was investigated. Moreover, the possibility of optimizing a GPR model for improving the retrieval accuracy of CRC was explored.

\subsubsection{Impact of Training Samples on ML Algorithm Performance}

ML algorithms typically require a large sample set to build an effective model. In order to analyze the effect of the number training samples on ML performance, each ML's ability to handle limited training samples was examined. The same training samples were used for each algorithm to compare ML performance against a matching set of variables. A uniform percentage of the total samples for each algorithm was chosen at twenty percent intervals starting at $40 \%$ and ending at $80 \%$. The sampling for each set was repeated 50 times. The models were trained with inputs of nine bands and four mean textures as an example. The results are presented in Table 7. Results demonstrate that with bands and texture measures as input variables, the best-performing model is SVR across all training sample size trials. The increase in training sample size improved the accuracy for CRC estimation, starting at $\overline{\mathrm{RMSE}_{\mathrm{CV}}}$ equal $6.544 \%$ for $40 \%$ training samples of the whole sample population and improving to $6.354 \%$ for an $80 \%$ training sample size. The $\overline{R_{c v}^{2}}$ increased with the increase in training samples. In general, all ML approaches improved accuracies with the increase in training sample size relative to total sample population.

Table 7. The accuracy for PLSR, ANN, GPR, SVR, and RF with nine bands and four mean textures as input variables for CRC estimation using varying percentages of the total samples $(n=243)$.

\begin{tabular}{|c|c|c|c|c|c|c|c|c|c|c|}
\hline \multirow{2}{*}{$\begin{array}{l}\text { Reference } \\
\text { Sample \% }\end{array}$} & \multicolumn{2}{|r|}{ PLSR } & \multicolumn{2}{|r|}{ ANN } & \multicolumn{2}{|r|}{ RF } & \multicolumn{2}{|r|}{ GPR } & \multicolumn{2}{|r|}{ SVR } \\
\hline & $\overline{R_{c v}^{2}}$ & $\overline{\operatorname{RMSE}_{\mathrm{cv}}}(\%)$ & $\overline{R_{c v}^{2}}$ & $\overline{\operatorname{RMSE}_{\mathrm{cv}}}(\%)$ & $\overline{R_{c v}^{2}}$ & $\overline{\operatorname{RMSE}_{\mathrm{cv}}}(\%)$ & $\overline{R_{c v}^{2}}$ & $\overline{\operatorname{RMSE}_{\mathrm{cv}}}(\%)$ & $\overline{R_{c v}^{2}}$ & $\overline{\operatorname{RMSE}_{\mathrm{cv}}}(\%)$ \\
\hline 40 & 0.59 & 7.088 & 0.57 & 8.619 & 0.58 & 7.228 & 0.61 & 6.838 & 0.64 & 6.544 \\
\hline 60 & 0.61 & 6.812 & 0.53 & 7.816 & 0.60 & 7.087 & 0.61 & 6.781 & 0.65 & 6.476 \\
\hline 80 & 0.62 & 6.769 & 0.60 & 7.690 & 0.62 & 6.739 & 0.63 & 6.731 & 0.66 & 6.354 \\
\hline
\end{tabular}

Note: $\overline{R_{c v}^{2}}$ and $\overline{\mathrm{RMSE}_{\mathrm{cv}}}(\%)$ are mean values of the cross-validation for 50 times randomly division of the whole samples into $40 \%, 60 \%$, and $80 \%$.

\subsubsection{Contribution of Input Predictors on ML Accuracy}

First, the five methods were trained and run with MSI bands as input features, yielding significantly higher accuracies than regressions based on individual bands. When the 13 CRIs from Table 1 were used as input features for a comparative analysis, the performances of the five models were further improved. However, regardless of whether nind MSI bands or 13 CRIs were used as input predictors for PLSR, ANN, GPR, and SVR, none of these models performed better than the univariate regression based on 3BI2 in terms of RMSE. When using mean textures and bands as input variable, the GPR, SVR, and RF models achieved better results than 3BI2. These three models also achieved a good performance by using mean textures and CRIs as predictors. Among the limited studies on the use of remote sensing indices as input features in ML models for the prediction of vegetation variables, Shah et al. [28] showed the possibility of using vegetation indices in the RF for estimating chlorophyll. 
Our results showed the potential for using CRIs and textural features together as input variables in ML approaches for the enhanced estimation of CRC. Moreover, these results indicated that input predictors have an impact on the performance of ML models and highlighted the importance of mean textural feature to obtain better estimates of CRC.

The PLSR is a powerful tool that can model several response variables simultaneously while effectively addressing strong collinear variables [52]. The retrieval accuracy of PLSR was improved by combining mean textures with bands. A combination of mean textures and CRIs via PLSR also enhanced the estimation of CRC. We extracted mean textural features from Sentinel-2 images, and correlated then with CRC. The results demonstrated that a combination of CRIs and mean textures via PLSR can enhance the retrieval accuracy of CRC.

ANN underperformed the other four ML models in terms of RMSE, regardless of the input features. This is likely to do with the fact that ANN is often used with large training datasets [53] and it does not perform well when used with input variables deviating from the small dataset presented during the training stage $[54,55]$. In this study, we had a limited size of training dataset $(n=243)$.

Recently, SVR gained popularity for the estimation of vegetation biophysical variables. Yang et al. [56] applied the SVR to estimate leaf area index and leaf chlorophyll density of rice, and found that SVR outperformed linear non-parametric methods. Our result showed that when bands or CRIs were used as input variables, SVR did not perform as well as GPR and RF. However, the SVR model based on a combination of bands and textures outperformed the other methods.

Among the four non-linear non-parametric techniques, GPR and RF have the ability to optimize input variables for the purpose of enhancing model simplicity and improved accuracy. We did not investigate the importance of input variables for RF model because many studies have already done so $[28,57]$. We emphasized the application of the $\sigma$ in the GPR covariance function to optimize input predictors. Our results demonstrate that the use of the optimal input variables filtered by $\sigma$ for each groups of variables enhanced the prediction accuracy of GPR. From Figure 7a, the MSI bands identified as highly important in the estimation of CRC were B3, B4, B5, B6, B11, and B12. When the nine MSI bands and four textural features were combined (Figure 7c), B12 was also identified as the optimal variable. B12 $(2190 \mathrm{~nm})$ is near $2100 \mathrm{~nm}$, where the reflectance of crop residue was mainly controlled by lignin and cellulose [58]. Moreover, indices in Table 1 associated with B12 provided a good retrieval of CRC (Table 3). It is likely that the selection of B12 can provide useful information about CRC. From Figure 7b, NDI5, NDRI, SGNDI, NDSVI, and 3BI1 were identified as the optimal input variables. It is worth noting that some of the CRIs that had high correlations with CRC were not identified as final input variables, such as 3BI2 and 3BI3, according to the values of $\sigma$. However, 3BI2 was selected as one of the optimal input variables when CRIs and textural features were combined (Figure 7d). Such a result agrees with the conclusion by Shah et al. [28] that remote sensing indices may perform variously when used in combination as input variables in a machine learner. Three of the four textural features were identified as optimal input variables not only when used in combination with bands but also when used in combination with CRIs (Figure 7c,d). The best input features for GPR were B4, B8a, B12, and mean textures of B3, B4, and B8. The results highlight the power of the GPR algorithms for identifying the optimal information for CRC estimation and the importance of selecting proper input variables for obtaining robust outputs.

\subsection{Importance of Textural Features for CRC Estimation}

Image texture is a quantification of the spatial variation of image tone values, which can be related to spatial distribution of vegetation [59]. The above-ground organization of crop residue elements is represented in texture, which is supplementary to the spectral image and may provide additional information about crop residue. In this study, although mean textural features extracted from Sentinel-2 10 bands have produced a low correlation with the UAV-derived CRC, we demonstrate that textural features have a high capability to provide an improvement in estimating CRC along with spectral information such as spectral bands and CRIs. For each ML algorithm, the combination of mean 
texture and MSI bands resulted in improvement in estimating CRC compared with MSI bands only. The combination of mean texture and CRIs also led to increasing estimation accuracy. Our experimental result confirmed Jin et al.'s findings [35]. They compared the power using eight textural measures extracted from Landsat 8 OLI bands to map CRC with CRIs based on PLSR. Their study showed that the combination of textural features and CRIs performed better than CRI-based variables for estimating CRC. Such similar conclusions suggest that textural information along with spectral information could potentially improve estimating CRC compared to using spectral information only. The benefits of combining textural measures and spectral information in estimating vegetation canopy parameters (i.e., LAI and vegetation fractional coverage) have been proved [32-34]. Zhou et al. [32] evaluated the performance of vegetation indices (VIs), texture measures, and combinations of VIs and texture measures on LAI estimation. They found that the approach based on a combination of VIs and textural feature yielded higher accuracy than the VI-based approach and texture-based approach. Based on these studies, it can be concluded that the accuracy of estimated variables based on remote-sensing data could be increased by considering textural information.

\subsection{Limitations of the Experiment}

We presented a low-cost method for mapping CRC at ultrahigh resolution in this study. UAV-derived RGB orthomosaics were classified into two classes: crop residue and background. Our results showed a high classification accuracy, which provides a good basis for training and evaluating Sentinel-2 data-based models. It should be noted that the inversion only relied on UAV-CRC data. Several studies demonstrated that the UAV orthomosaics interpretation enables the cost-effective creation of large and comprehensive datasets in comparison to field work [60,61]; therefore, field measures of CRC were not included in this paper, given the ultrahigh resolution of UAV data. We acknowledge that there is space for improvement in the classification accuracy by comparing different classification methods [62], such as object-based image analysis, or logistic regression, which is a powerful statistical learning method for a two-class situation [63].

\section{Conclusions}

In this study, we focused on the estimation of CRC based on empirical regressions and machine learning methods from Sentinel-2 imagery. Based on UAV collected CRC and near-synchronous Sentinel-2 image, inversion models were established and validated. The results show that 3BI1, 3BI2, and 3BI3 improved the sensitivity to CRC and the estimation accuracy compared to the published CRIs. MSI bands, CRIs, and their combinations with mean textural features were used as input variables for PLSR, ANN, GPR, SVR and RF models. The five ML approaches with bands as input variables yielded accuracies second to those methods based on CRIs, the combination of indices and textural features, and the combination of bands and textural features, respectively. The SVR with bands and textural features yielded excellent performance $\left(R_{\mathrm{cv}}^{2}=0.67, \mathrm{RMSE}_{\mathrm{cv}}=6.343 \%\right)$. The GPR based on CRIs and textural features also obtained high accuracy $\left(R_{\mathrm{cv}}^{2}=0.66, \mathrm{RMSE}_{\mathrm{cv}}=6.352 \%\right)$. The retrieval accuracy of each GPR model optimized by $\sigma$ was further improved. The best-performing model was a GPR with input variables of red, NIR, the second SWIR bands, and mean textural features of blue, red, and NIR bands, obtaining an accuracy of $R^{2}$ cv equal 0.69 and $R M S E_{c v}$ equal $6.149 \%$. Comparing empirical regression and machine learning methods, it can be concluded that (1) most of the five ML models with the optimal input variables performed better than univariate regression, (2) using a scheme of combining mean textural features with spectral information could lead to higher accuracy of estimating CRC than spectral information alone, and (3) GPR is recommended for CRC estimation.

Supplementary Materials: The hyperparameters for ML models and the 10-fold cross-validation result of each fold for each algorithm. The following are available online at http://www.mdpi.com/2072-4292/12/9/1470/s1, Table S1: Optimized hyperparameters for ANN with 9 bands and 4 mean textural features as input predictors. Table S2: Optimized hyperparameters for SVR with 9 bands and 4 mean textural features as input predictors. Table S3: Optimized hyperparameters for RF with 9 bands and 4 mean textural features as input predictors. Table S4: Optimized hyperparameters for GPR with 9 bands and 4 mean textural features as input predictors. 
Table S5: The $R^{2}$ cv and $\mathrm{RMSE}_{\mathrm{cv}}(\%)$ values for each fold of the 10 -fold cross-validation of the univariate regressions based on MSI bands. Table S6: The continuation of Table S5. Table S7: The $R^{2}{ }_{\mathrm{cv}}$ and $\mathrm{RMSE}_{\mathrm{cv}}(\%)$ values for each fold of the 10-fold cross-validation of the univariate regressions based on CRIs. Table S8: The continuation of Table S7. Table S9: The continuation of Table S8. Table S10: The $R^{2}{ }_{\mathrm{cv}}$ and $\mathrm{RMSE}_{\mathrm{cv}}(\%)$ values for each fold of the 10 -fold cross-validation of the univariate regressions based on textural features. Table S11: $R^{2}{ }_{\mathrm{cv}}$ and RMSEcv (\%) values for each fold of the 10-fold cross-validation of the five ML approaches with 9 bands and 4 mean textural features as input predictors.

Author Contributions: Conceptualization, Y.D., H.Z. and Y.W.; Data curation, L.L.; Formal analysis, Y.D.; Funding acquisition, Y.D.; Methodology, Y.D. and Y.W.; Writing-original draft, Y.D.; Writing-review and editing, Z.W., Q.X. Y.W., and C.C.H. All authors have read and agreed to the published version of the manuscript.

Funding: This research was funded by the National Key Research and Development Program of China, grant number 2016YFD0200102, the Technology Development Program of Jilin Province, China, grant number 20180201012GX, “The 13th Five-Year plan" Science and Technology Project of the Department of Education, Jilin Province, grant number JJKH20170915KJ, and the Jilin Province Science and Technology Development Plan, grant number $20190301057 \mathrm{NY}$.

Acknowledgments: The authors would like to extend their appreciation to Xingming Zheng and Tao Jiang of Northeast Institute of Geography and Agroecology, Chinese Academy of Sciences and their laboratory staff for their support to collecting UAV images. The authors wish to thank the three anonymous reviewers and the academic editor for their constructive comments which improved the manuscript.

Conflicts of Interest: The authors declare no conflict of interest.

\section{References}

1. Haddaway, N.R.; Hedlund, K.; Jackson, L.E.; Kätterer, T.; Lugato, E.; Thomsen, I.K.; Jørgensen, H.B.; Isberg, P.-E. How does tillage intensity affect soil organic carbon? A systematic review. Environ. Evid. 2017, 6, 30. [CrossRef]

2. R, H.P.; Ken, S.; Raj, G. The role of conservation agriculture in sustainable agriculture. Philos. Trans. R. Soc. 2008, 363, 543-555.

3. Farina, R.; Seddaiu, G.; Orsini, R.; Steglich, E.; Roggero, P.P.; Francaviglia, R. Soil carbon dynamics and crop productivity as influenced by climate change in a rainfed cereal system under contrasting tillage using epic. Soil Till. Res. 2011, 112, 36-46. [CrossRef]

4. Cai, W.; Zhao, S.; Wang, Y.; Peng, F.; Heo, J.; Duan, Z. Estimation of winter wheat residue coverage using optical and SAR remote sensing images. Remote Sens. 2019, 11, 1163. [CrossRef]

5. Morrison, J.E.; Huang, C.-H.; Lightle, D.T.; Daughtry, C.S. Residue measurement techniques. J. Soil Water Conserv. 1993, 48, 478-483.

6. Baker, N. Tillage Practices in the Conterminous United States, 1989-2004-Datasets Aggregated by Watershed; Data Series 573; US Geological Survey: Reston, VA, USA, 2011. Available online: http://pubs.usgs.gov/ds/ds573 (accessed on 3 April 2020).

7. Zheng, B.; Campbell, J.B.; Serbin, G.; Galbraith, J.M. Remote sensing of crop residue and tillage practices: Present capabilities and future prospects. Soil Till. Res. 2014, 138, 26-34. [CrossRef]

8. Hively, W.D.; Shermeyer, J.; Lamb., B.T.; Daughtry, C.S.; Quemada, M. Mapping crop residue by combining Landsat and Worldview 3 satellite imagery. Remote Sens. 2019, 11, 1857. [CrossRef]

9. Sudheer, K.; Gowda, P.; Chaubey, I.; Howell, T. Artificial neural network approach for mapping contrasting tillage practices. Remote Sens. 2010, 2, 579-590. [CrossRef]

10. Watts, J.D.; Powell, S.L.; Lawrence, R.L.; Hilker, T. Improved classification of conservation tillage adoption using high temporal and synthetic satellite imagery. Remote Sens. Environ. 2011, 115, 66-75. [CrossRef]

11. Pacheco, A.; McNairn, H. Evaluating multispectral remote sensing and spectral unmixing analysis for crop residue mapping. Remote Sens. Environ. 2010, 114, 2219-2228. [CrossRef]

12. Biard, F.; Baret, F. Crop residue estimation using multiband reflectance. Remote Sens. Environ. 1997, 59, 530-536. [CrossRef]

13. Yue, J.; Tian, Q.; Dong, X.; Xu, K.; Zhou, C. Using hyperspectral crop residue angle index to estimate maize and winter-wheat residue cover: a laboratory study. Remote Sens. 2019, 11, 807. [CrossRef]

14. Nagler, P.; Daughtry, C.; Goward, S. Plant litter and soil reflectance. Remote Sens. Environ. 2000, 71, $207-215$. [CrossRef] 
15. Serbin, G.; Daughtry, C.S.T.; Hunt, E.R.; Brown, D.J.; McCarty, G.W. Effect of soil spectral properties on remote sensing of crop residue cover. Soil Sci. Soc. Am. J. 2009, 73, 1545. [CrossRef]

16. Serbin, G.; Hunt, E.R.; Daughtry, C.S.; McCarty, G.; Doraiswamy, P. An improved ASTER index for remote sensing of crop residue. Remote Sens. 2009, 1, 971-991. [CrossRef]

17. Sullivan, D.G.; Truman, C.C.; Schomberg, H.H.; Endale, D.M.; Strickland, T.C. Evaluating techniques for determining tillage regime in the Southeastern Coastal Plain and Piedmont. Agron J. 2006, 98, 1236-1246. [CrossRef]

18. Quemada, M.; Daughtry, C. Spectral indices to improve crop residue cover estimation under varying moisture conditions. Remote Sens. 2016, 8, 660. [CrossRef]

19. Van Deventer, A.; Ward, A.; Gowda, P.; Lyon, J. Using thematic mapper data to identify contrasting soil plains and tillage practices. Photogramm. Eng. Rem. S. 1997, 63, 87-93.

20. McNairn, H.; Protz, R. Mapping corn residue cover on agricultural fields in Oxford County, Ontario, using thematic mapper. Can. J. Remote Sens. 1993, 19, 152-159. [CrossRef]

21. Qi, J.; Marsett, R.; Heilman, P.; Bieden-bender, S.; Moran, S.; Goodrich, D.; Weltz, M. RANGES improves satellite-based information and land cover assessments in southwest United States. Eos Trans. Am. Geophys. Union 2002, 83, 601-606. [CrossRef]

22. Gelder, B.; Kaleita, A.; Cruse, R. Estimating mean field residue cover on midwestern soils using satellite imagery. Agron. J. 2009, 101, 635-643. [CrossRef]

23. Verrelst, J.; Rivera, J.P.; Veroustraete, F.; Muñoz-Marí, J.; Clevers, J.G.P.W.; Camps-Valls, G.; Moreno, J. Experimental Sentinel-2 LAI estimation using parametric, non-parametric and physical retrieval methods-A comparison. ISPRS J. Photogramm. 2015, 108, 260-272. [CrossRef]

24. Yue, J.; Tian, Q.; Dong, X.; Xu, N. Using broadband crop residue angle index to estimate the fractional cover of vegetation, crop residue, and bare soil in cropland systems. Remote Sens. Environ. 2020, 237, 111538. [CrossRef]

25. Verrelst, J.; Camps-Valls, G.; Muñoz-Marí, J.; Rivera, J.P.; Veroustraete, F.; Clevers, J.G.P.W.; Moreno, J. Optical remote sensing and the retrieval of terrestrial vegetation bio-geophysical properties-A review. ISPRS J. Photogramm. 2015, 108, 273-290. [CrossRef]

26. Lary, D.J.; Alavi, A.H.; Gandomi, A.H.; Walker, A.L. Machine learning in geosciences and remote sensing. Geosci. Front. 2016, 7, 3-10. [CrossRef]

27. Jia, K.; Liang, S.L.; Liu, S.H.; Li, Y.W.; Xiao, Z.Q.; Yao, Y.J.; Jiang, B.; Zhao, X.; Wang, X.X.; Xu, S.; et al. Global land surface fractional vegetation cover estimation using general regression neural networks from modis surface reflectance. IEEE Trans. Geosci. Remote 2015, 53, 4787-4796. [CrossRef]

28. Shah, S.H.; Angel, Y.; Houborg, R.; Ali, S.; McCabe, M.F. A random forest machine learning approach for the retrieval of leaf chlorophyll content in wheat. Remote Sens. 2019, 11, 920. [CrossRef]

29. Yin, G.; Verger, A.; Qu, Y.; Zhao, W.; Xu, B.; Zeng, Y.; Liu, K.; Li, J.; Liu, Q. Retrieval of high spatiotemporal resolution leaf area index with gaussian processes, wireless sensor network, and satellite data fusion. Remote Sens. 2019, 11, 244. [CrossRef]

30. Bocco, M.; Sayago, S.; Willington, E. Neural network and crop residue index multiband models for estimating crop residue cover from Landsat tm and ETM+ images. Int. J. Remote Sens. 2014, 35, 3651-3663. [CrossRef]

31. Daughtry, C.S.T.; Doraiswamy, P.C.; Hunt, E.R.; Stern, A.J.; McMurtrey, J.E.; Prueger, J.H. Remote sensing of crop residue cover and soil tillage intensity. Soil Till. Res. 2006, 91, 101-108. [CrossRef]

32. Zhou, J.; Zhao, Z.; Zhao, J.; Zhao, Q.; Wang, F.; Wang, H. A comparison of three methods for estimating the LAI of black locust (Robinia pseudoacacia L.) plantations on the Loess Plateau, China. Int. J. Remote Sens. 2014, 35, 171-188. [CrossRef]

33. Shamsoddini, A.; Trinder, J.C.; Turner, R. Pine plantation structure mapping using WorldView-2 multispectral image. Int. J. Remote Sens. 2013, 34, 3986-4007. [CrossRef]

34. Pu, R.; Cheng, J. Mapping forest leaf area index using reflectance and textural information derived from WorldView-2 imagery in a mixed natural forest area in Florida, US. Int. J. Appl. Earth Obs. Geoinf. 2015, 42, 11-23. [CrossRef]

35. Jin, X.; Ma, J.; Wen, Z.; Song, K. Estimation of maize residue cover using Landsat-8 OLI image spectral information and textural features. Remote Sens. 2015, 7, 14559-14575. [CrossRef]

36. Mountrakis, G.; Im, J.; Ogole, C. Support vector machines in remote sensing: A review. ISPRS J. Photogramm. 2011, 66, 247-259. [CrossRef] 
37. Shao, Y.; Lunetta, R.S. Comparison of support vector machine, neural network, and cart algorithms for the land-cover classification using limited training data points. ISPRS J. Photogramm. 2012, 70, 78-87. [CrossRef]

38. Cao, X.; Chen, J.; Matsushita, B.; Imura, H. Developing a MODIS-based index to discriminate dead fuel from photosynthetic vegetation and soil background in the Asian steppe area. Int. J. Remote Sens. 2010, 31, 1589-1604. [CrossRef]

39. Daughtry, C.; Hunt, E.; Doraiswamy, P.; McMurtrey, J. Remote sensing the spatial distribution of crop residues. Agron. J. 2005, 97, 864-871. [CrossRef]

40. Sullivan, D.; Lee, D.; Beasley, J.; Brown, S.; Williams, E. Evaluating a crop residue cover index for determining tillage regime in a cotton-corn-peanut rotation. J. Soil Water Conserv. 2008, 63, 28-36. [CrossRef]

41. Hansen, P.M.; Schjoerring, J.K. Reflectance measurement of canopy biomass and nitrogen status in wheat crops using normalized difference vegetation indices and partial least squares regression. Remote Sens. Environ. 2003, 86, 542-553. [CrossRef]

42. Baret, F.; Weiss, M.; Lacaze, R.; Camacho, F.; Makhmara, H.; Pacholcyzk, P.; Smets, B. GEOV1: LAI and FAPAR essential climate variables and fcover global time series capitalizing over existing products. Part1: Principles of development and production. Remote Sens. Environ. 2013, 137, 299-309. [CrossRef]

43. Hagan, M.T.; Menhaj, M.B. Training feedforward networks with the marquardt algorithm. IEEE Trans. Neural Netw. 1994, 5, 989-993. [CrossRef] [PubMed]

44. Vapnik, V.; Golowich, S.E.; Smola, A.J. Support vector method for function approximation, regression estimation and signal processing. Adv. Neural Inform. Process. Syst. 1997, 9, 281-287.

45. Andrew, A.M. An introduction to support vector machines and other kernel-based learning methods. Kybernetes 2001, 30, 103-115.

46. Rasmussen, C.E. Gaussian Processes in Machine Learning; The MIT Press: New York, NY, USA, 2006.

47. Hultquist, C.; Chen, G.; Zhao, K. A comparison of gaussian process regression, random forests and support vector regression for burn severity assessment in diseased forests. Remote Sens. Lett. 2014, 5, 723-732. [CrossRef]

48. Breiman, L. Random forests. Mach. Learn. 2001, 45, 5-32. [CrossRef]

49. Belgiu, M.; Drăguţ, L. Random forest in remote sensing: A review of applications and future directions. ISPRS J. Photogramm. 2016, 114, 24-31. [CrossRef]

50. Meyer, H.; Lehnert, L.W.; Wang, Y.; Reudenbach, C.; Nauss, T.; Bendix, J. From local spectral measurements to maps of vegetation cover and biomass on the qinghai-tibet-plateau: Do we need hyperspectral information? Int. J. Appl. Earth Obs. Geoinf. 2017, 55, 21-31. [CrossRef]

51. Verrelst, J.; Alonso, L.; Camps-Valls, G.; Delegido, J.; Moreno, J. Retrieval of vegetation biophysical parameters using gaussian process techniques. IEEE Trans. Geosci. Remote 2012, 50, 1832-1843. [CrossRef]

52. Li, X.L.; Zhang, Y.J.; Luo, J.H.; Jin, X.L.; Xu, Y.; Yang, W.Z. Quantification winter wheat LAI with HJ-1 CCD image features over multiple growing seasons. Int. J. Appl. Earth Obs. Geoinf. 2016, 44, 104-112. [CrossRef]

53. Wang, L.a.; Zhou, X.; Zhu, X.; Dong, Z.; Guo, W. Estimation of biomass in wheat using random forest regression algorithm and remote sensing data. Crop J. 2016, 4, 212-219. [CrossRef]

54. Atzberger, C. Object-based retrieval of biophysical canopy variables usingartificial neural nets and radiative transfer models. Remote Sens. Environ. 2004, 93, 53-67. [CrossRef]

55. Kimes, D.S.; Nelson, R.F.; Manry, M.T.; Fung, A.K. Attributes of neural networks for extracting continuous vegetation variables from optical and radar measurements. Int. J. Remote Sens. 1998, 19, 2639-2662. [CrossRef]

56. Yang, X.H.; Huang, J.F.; Wu, Y.P.; Wang, J.W.; Wang, p.; Wang, X.M.; Huete, A. Estimating biophysical parameters of rice with remote sensing data using support vector machines. Sci. China Life Sci. 2011, 54, 272-281. [CrossRef] [PubMed]

57. Mutanga, O.; Adam, E.; Cho, M.A. High density biomass estimation for wetland vegetation using WorldView-2 imagery and random forest regression algorithm. Int. J. Appl. Earth Obs. Geoinf. 2012, 18, 399-406. [CrossRef]

58. Daughtry, C.S.T.; Hunt, E.R.; McMurtrey, J.E., III. Assessing crop residue cover using shortwave infrared reflectance. Remote Sens. Environ. 2004, 90, 126-134. [CrossRef]

59. Colombo, R.; Bellingeri, D.; Fasolini, D.; Marino, C.M. Retrieval of leaf area index in different vegetation types using high resolution satellite data. Remote Sens. Environ. 2003, 86, 120-131. [CrossRef]

60. Zhujun, G.; Ju, W.; Li, L.; Li, D.; Liu, Y.; Fan, W. Using vegetation indices and texture measures to estimate vegetation fractional coverage (VFC) of planted and natural forests in Nanjing city, China. Adv. Space Res. 2013, 51, 1186-1194. 
61. Riihimäki, H.; Luoto, M.; Heiskanen, J. Estimating fractional cover of tundra vegetation at multiple scales using unmanned aerial systems and optical satellite data. Remote Sens. Environ. 2019, 224, 119-132.

62. Cai, Y.T.; Zhang, M.; Lin, H. Estimating the urban fractional vegetation cover using an object-based mixture analysis method and Sentinel-2 MSI imagery. IEEE J.-STARS 2020, 13, 341-350. [CrossRef]

63. Blaschke, T.; Hay, G.J.; Kelly, M.; Lang, S.; Hofmann, P.; Addink, E.; Feitosa, R.Q.; van der Meer, F.; van der Werff, H.; van Coillie, F.; et al. Geographic object-based image analysis-towards a new paradigm. ISPRS J. Photogramm. 2014, 87, 180-191. [CrossRef] [PubMed]

(C) 2020 by the authors. Licensee MDPI, Basel, Switzerland. This article is an open access article distributed under the terms and conditions of the Creative Commons Attribution (CC BY) license (http://creativecommons.org/licenses/by/4.0/). 